\title{
A 12-bit 100kS/s SAR ADC for Biomedical Applications
}

\author{
Sung-Chan Rho ${ }^{1}$ and Shin-Il Lim ${ }^{2 *}$ \\ ${ }^{1}$ Department of Electronics and Computer Engineering, Seokyeong University \\ ${ }^{2}$ Department of Electronics Engineering, Seokyeong University \\ Seoul, Korea \\ ${ }^{1}$ scrho@skuniv.ac.kr, ${ }^{2}$ silim@skuniv.ac.kr
}

\begin{abstract}
This paper describes a 12-bit 100kS/s successive approximation register analog-todigital converter (SAR ADC) for biomedical system. Both top-plate sampling technique and VCM-based switching technique are applied to the capacitor digital-to-analog converter $(C D A C)$ to implement a 12-bit SAR ADC with 10-b capacitor array DAC. To enhance the linearity of proposed $A D C$, thermometer decoder is used in capacitor array DAC. Switching-energy minimization technique, asynchronous control with a low-power delay circuit and true single phase clocking (TSPC) D_FF are also adopted to reduce power consumption. Simulation results show that the proposed ADC achieves the SNDR of $70.97 \mathrm{~dB}$, the SDFR of $80.23 \mathrm{~dB}$ and the ENOB of $11.49 \mathrm{~b}$ with the CMOS $0.18 \mu \mathrm{m}$ technology. Total power consumption is $11.16 \mu \mathrm{W}$ under the supply voltage of $1.8 \mathrm{~V}$ at the sampling frequency of $100 \mathrm{kHz}$. And the figure of merit (FoM) is 38.79fJ/conversionsteps.
\end{abstract}

Keywords: Analog-to-digital converter (ADC), SAR ADC, thermometer decoder DAC, dummy Cap switching, energy-efficient, biomedical system

\section{Introduction}

Due to the good power efficiency, the SAR type analog-to-digital converter (ADC) is widely used in biomedical system [1-3]. In biomedical applications such as electrocardiogram (ECG) and electro-encephalogram (EEG), the resolution of ADC over 10-bit to 12-bit is required for high accuracy in analog front end. Since the SAR ADC is substantially implemented with the capacitor array in internal DAC, the capacitor array in 12-bit DAC requires large chip area if it is implemented with direct binary weighted capacitor array. And as the recent biomedical devices are implemented with portable form, low power consumption is essential design condition for battery operated system. In accordance with this trend, this paper focuses on the implementation of 12-bit SAR type ADC with low power consumption and small chip area.

The organization of this paper is as follows: section 2 presents the architecture of the proposed 12-bit SAR ADC, section 3 describes the details of the proposed 12-bit SAR ADC with 10-bit DAC, low power delay circuits [4], section 4 reports the simulation results and performance summary, and finally the conclusion is in section 5 .

\section{Architecture of the Proposed ADC}

Figure 1 shows the block diagram of proposed 12-bit SAR ADC. It has differential charge redistribution DAC, an output offset cancelled comparator, a SAR and an asynchronous control block. The proposed 12-bit SAR ADC used top plate sampling technique [5] and also dummy capacitor switching technique based on common mode

${ }^{*}$ Corresponding Author 
voltage (VCM) [6] to reduce the chip area and power consumption. Split capacitor arrays with attenuation capacitor in differential DAC are also adopted to reduce hardware as shown in Figure 2. In addition, for the enhanced accuracy, output offset cancelled comparator and thermometer decoder DAC is used. And, for the low power consumption in asynchronous digital control block, leakage based low power delay circuit is adopted.

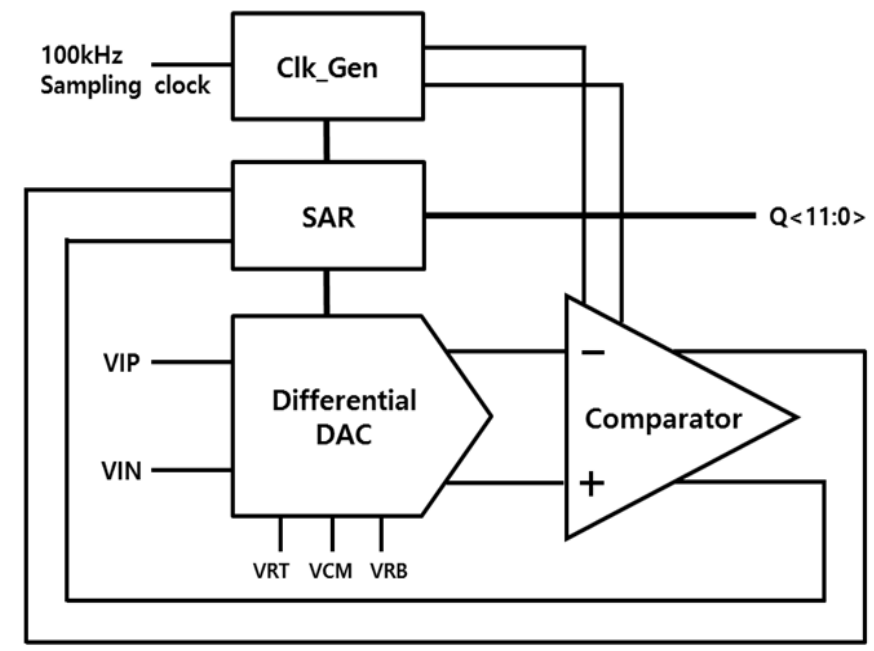

Figure 1. Block Diagram of the 12-bit SAR ADC

\section{Proposed ADC}

\subsection{Proposed DAC}

Figure 2, shows the architecture of 10-bit DAC in 12-bit ADC. The thermometer decoder is applied to the MSB array in DAC to improve the linearity of the SAR ADC. In sampling mode, input signal is applied to the top plate of differential capacitor array. In holding mode, DAC holds VIN and VIP. Since the MSB value is determined by comparing the initial holding values of differential DAC, the MSB capacitor array in conventional DAC is not needed. This technique enables to reduce the total capacitance by half compared to the conventional design. By switching the reference voltage on the last unit capacitor (dummy capacitor) between (VRT, VCM) instead of (VRT, VRB), additional LSB comparison is allowed. This dummy capacitor switching technique based on VCM in LSB decision could reduce the lower sub-capacitor array to another half. As a result, this $\mathrm{V}_{\mathrm{CM}}$-based LSB switching technique [6], together with aforementioned top-plate sampling technique [5], allowed us to implement a 12-bit ADC with a 10-bit capacitor array DAC.

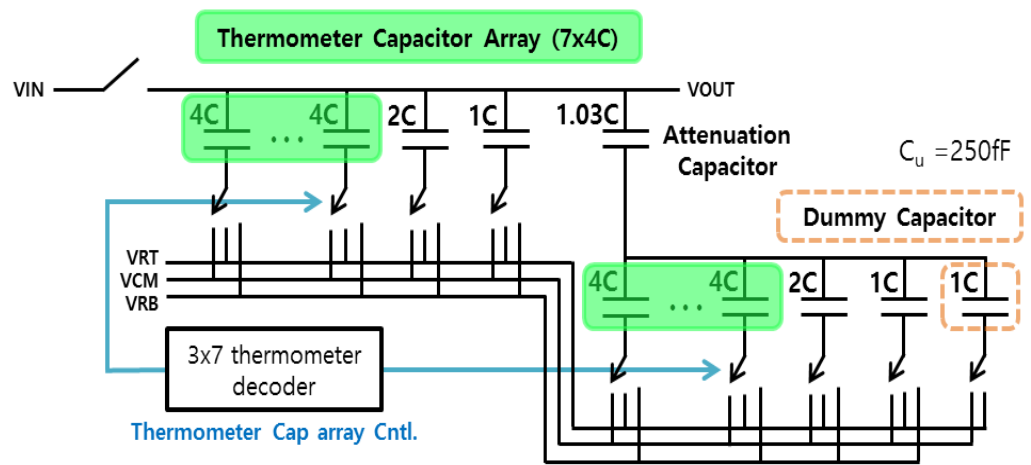

Figure 2. Diagram of the Proposed 10-Bit DAC in 12-Bit ADC 


\subsection{Dummy Capacitor Switching Technique}

Figure 3, (a) shows the conventional switching process of 3-bit DAC as an example. In the sampling periods, both VIP and VIN are sampled to the bottom plate of capacitor arrays. During the 1st conversion process in holding periods, the MSB capacitors are switched to voltage reference top (VRT) and other capacitors are connected to voltage reference bottom (VRB) in the upper array of DAC. And reverse reference voltages are also connected in the lower array of DAC.

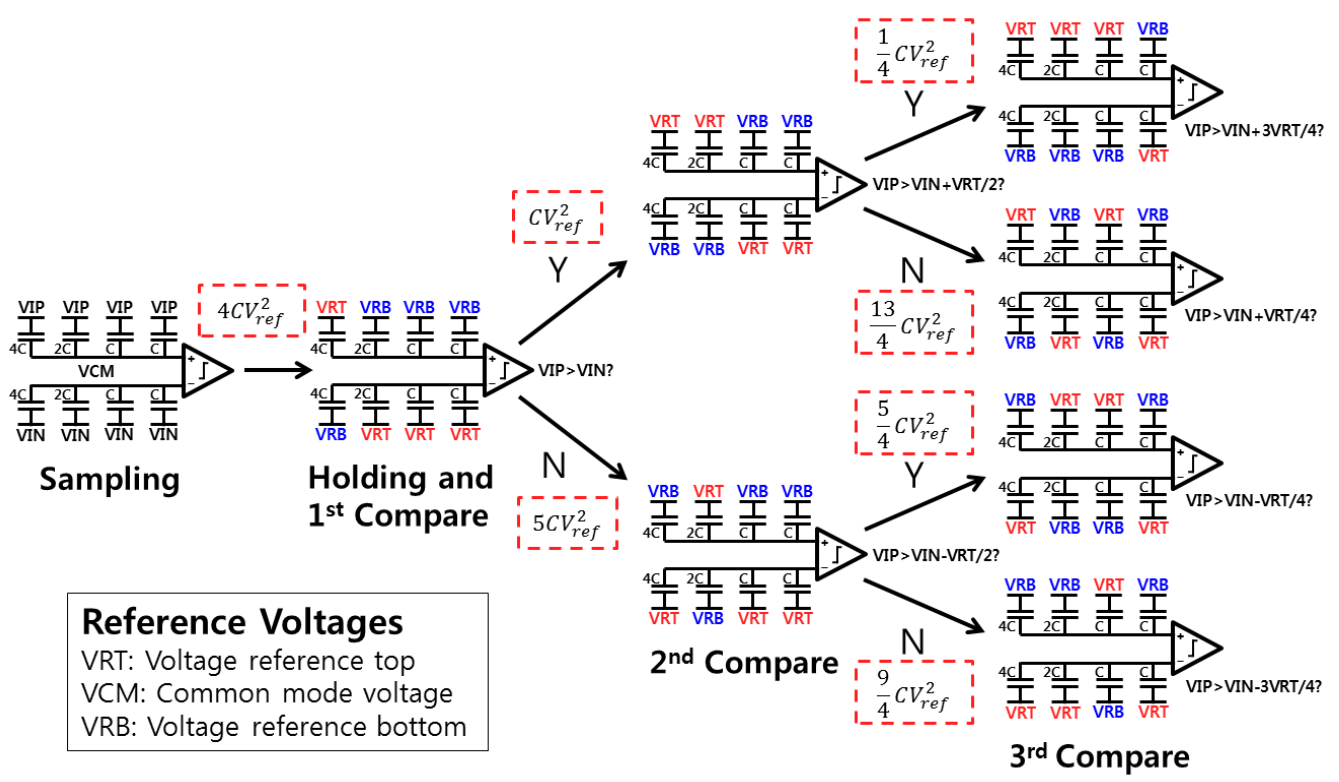

(a)

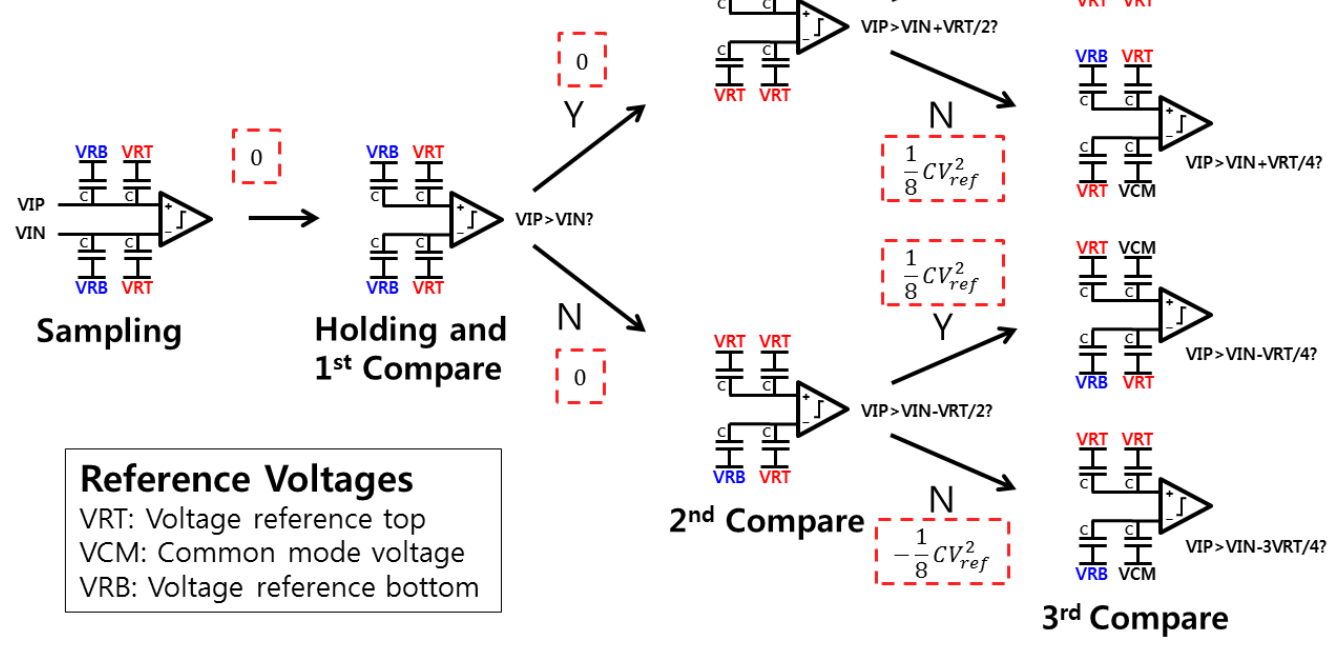

(b)

Figure 3. (a) Conventional Switching Process with 3b DAC Example (b) Implementation of the Low Switching Energy Process with 3b DAC Example 
In this case, all the capacitors switched the reference voltage and hence consume the switching power of $4 \mathrm{CV}^{2}$. [7] Also, during the $2^{\text {nd }}$ and $3^{\text {rd }}$ conversion process in holding period, this conventional switching technique consumes more switching energy as shown Figure 3, (a).

Figure 3, (b) shows the process of proposed low switching energy technique with 3-bit DAC as a conceptual example. Capacitors in proposed DAC are one quarter of capacitors in conventional DAC as mentioned earlier. If both VIP and VIN are sampled to the top plate of each capacitor array, there we have zero switching energy during $1^{\text {st }}$ conversion. Also for the remaining LSB decisions, we need zero switching energy in $2^{\text {nd }}$ conversion cycle and $1 / 8 \mathrm{CV}^{2}$ in $3^{\text {rd }}$ conversion cycle, as shown Figure 3, (b).

\subsection{Low Power Delay Circuits}

Figure 4(a) shows the delay circuits [4] in asynchronous control block for low power consumption. In conventional delay circuits with inverters and load capacitors, a large amount of static current flows. However, the adopted delay circuits make the longer delay time without large static currents, because its leakage-based large resistance of MOS transistors and gate capacitance generate the longer RC delay as shown in Figure 4, (b). It consumes a current of $400 \mathrm{nA}$ when the delay circuit generates the delay of $200 \mathrm{~ns}$.

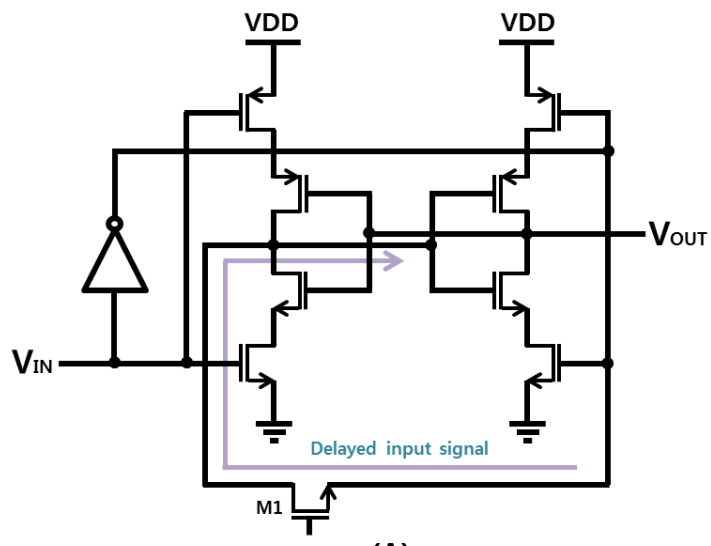

(A)

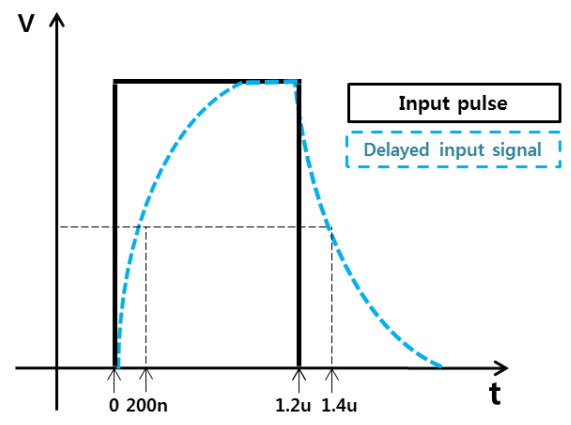

(B)

Figure 4. (a) Implementation of the Low Power Delay Circuits (b) Input Pulse and Output Pulse with the Delay of 200ns

\section{Simulation Results and Performance Summary}

The chip was implemented with a $0.18 \mu \mathrm{m}$ CMOS technology. The core area, as shown in Figure 6, is $877 \mu \mathrm{m} \times 479 \mu \mathrm{m}$, excluding pads. The FFT simulation results with the 21 $\mathrm{kHz}$ input signal at sampling rate of $100 \mathrm{kHz}$ are shown in Figure 7. The proposed ADC achieves the SNDR of $70.97 \mathrm{~dB}$, the SFDR of $80.23 \mathrm{~dB}$ and the ENOB of $11.49 \mathrm{bits}$. The power consumes $11.16 \mu \mathrm{W}$ at a sampling frequency of $100 \mathrm{kHz}$ under supply voltage of $1.8 \mathrm{~V}$. And the figure of merit $(\mathrm{FoM})$ is $38.49 \mathrm{fJ} /$ conversion-steps. The performance is summarized in Table 1. 


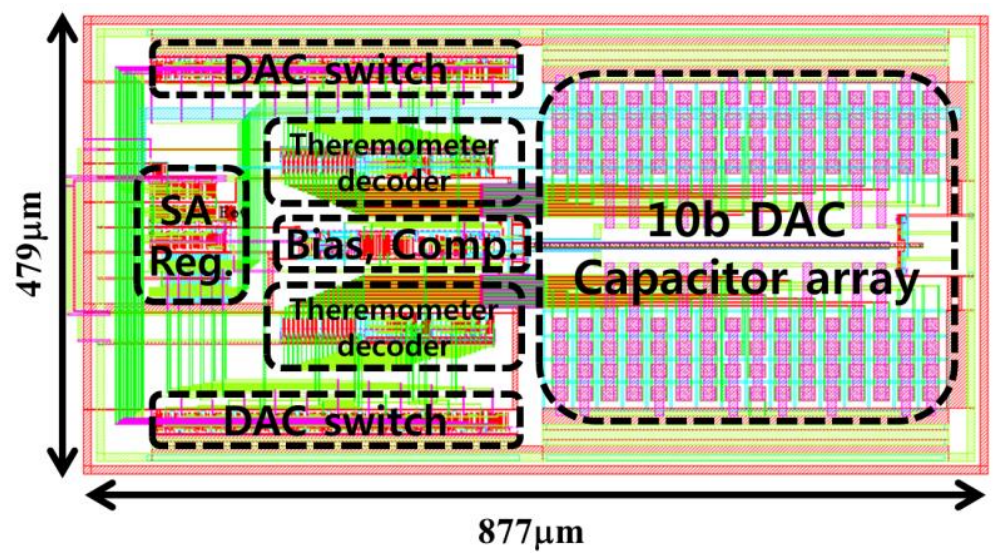

Figure 6. Chip Layout (w/o Pad)

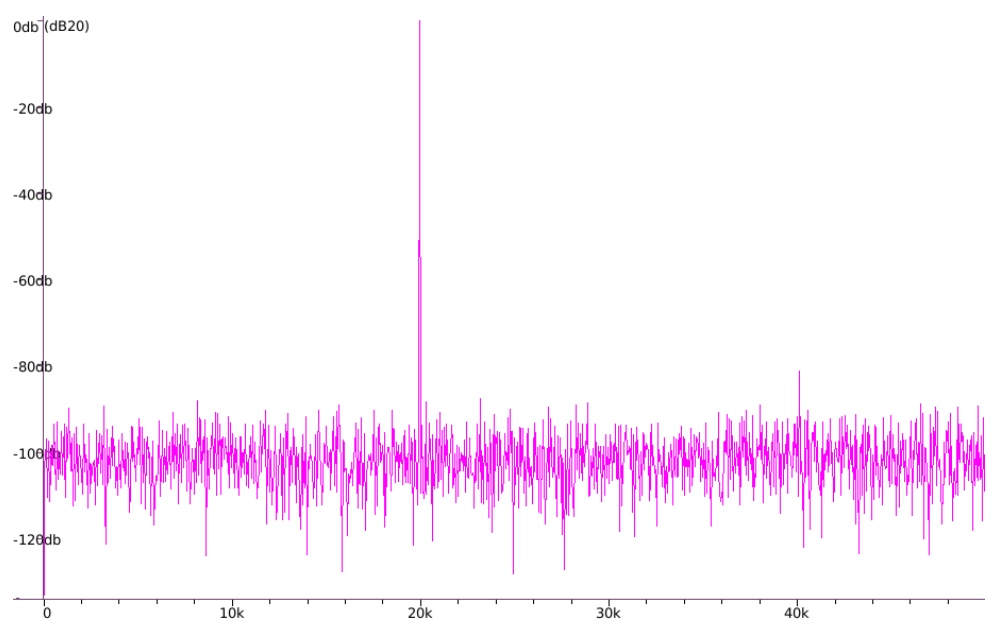

Figure 7. FFT Simulation Results

Table 1. Performance Summary

\begin{tabular}{|c|c|}
\hline Technology & Magna 0.18 $\mu \mathrm{m}$ CMOS \\
\hline Resolution & $12-$ bit \\
\hline Power supply & $1.8 \mathrm{~V}$ \\
\hline Sampling rate & $100 \mathrm{kHz}$ \\
\hline FoM & $\mathbf{3 8 . 4 9 f J} /$ conv. \\
\hline SNDR, SFDR & $\mathbf{7 0 . 9 7 d B , 8 0 . 2 3 d B}$ \\
\hline ENOB & 11.49 -bit \\
\hline Layout & $877 \mu \mathrm{m} \times 479 \mu \mathrm{m}$ \\
\hline
\end{tabular}

\section{Conclusion}

The 12-bit 100kS/s SAR ADC was designed with both a top-plate sampling technique and a VCM-based switching technique to reduce chip area and also to reduce power consumption. Additionally, for further reducing power consumption, a leakage-based delay circuit and TSPC D_FF are used. The thermometer decoder applied to the MSB arrays in DAC improves the linearity of the SAR ADC. 


\section{Acknowledgments}

This research was supported by the Industrial Core Technology Development Program (10049009) funded by the Ministry of Trade, Industry \& Energy (MITIE), Korea and also supported by the MSIP(Ministry of Science, ICT and Future Planning), Korea, under the ITRC(Information Technology Research Center) support program (IITP-2016-H8501-161010) supervised by the IITP(Institute for Information \& communications Technology Promotion). The CAD tools were supported by IC design Education Center (IDEC).

\section{References}

[1] G. Y. Huang, S. J. Chang, C. C. Liu and Y. Z. Lin, "A 1- $\mu \mathrm{m}$ W 10-bit 200-kS/s SAR ADC with a bypass window for biomedical applications," IEEE J. Solid-State Circuits, vol. 47, no. 11, (2012) November, pp. 2783-2795.

[2] D. Zhang, A. Bhide and A. Alvandpour, "A 53-nW 9.1-ENOB 1-kS/s SAR ADC in 0.13- $\mu \mathrm{m}$ CMOS for medical implant devices," IEEE J. Solid-State Circuits, vol. 47, no. 7, (2012) July, pp. 1585-1593.

[3] S. Yan, X. Zhongming and G. Li, "A 0.6-V 8.3-ENOB asynchronous SAR ADC for biomedical applications", Journal of semiconductors, vol. 35, no. 8, (2014) August.

[4] W. Jung, S. Oh, S. Bang, Y. Lee, D. Sylvester and D. Blaauw, "A $3 \mathrm{nW}$ fully integrated energy harvester based on self-oscillating switched-capacitor DC-DC converter", IEEE ISSCC Dig. Tech. Paper August, pp. 398 -399.

[5] Y. Z. Lin, C.-C. Liu, G.-Y. Huang, Y.-T. Shyu and S. J. Chang, "A 9-bit 150-MS/s 1.53-mW subranged SAR ADC in 90-nm CMOS", IEEE Symp. VLSI Circuits Dig., (2010) June, pp. 243-244,

[6] A. Sanyal and N. Sun, "An energy-efficient. low frequency-dependence switching technique for SAR ADCs", IEEE Trans. Circuits Syst.-II, vol. 61, no. 5, (2014) February, pp. 294 -298.

[7] B. P. Ginsburg and A. P. Chandrakasan, "An energy-efficient charge recycling approach for a SAR converter with capacitive DAC”, IEEE Symp. VLSI Circuits Dig., (2005) May, pp. 184 -187.

\section{Authors}

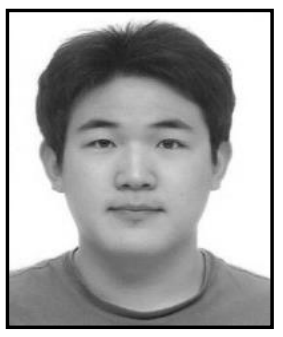

Sung-Chan Rho received B.S. degrees in the Department of Electronics Engineering from Seokyeong University, Seoul, Korea, in 2015. Since 2015, he has been taking his master course in Seokyeong University. His research interests include analog and mixed mode IC design for biomedical and sensor applications.

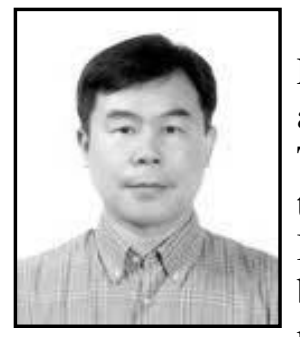

Shin-Il Lim received his BS, MS and $\mathrm{PhD}$ degrees in Electronic Engineering from Sogang University, Seoul, Korea, in 1980, 1983, and 1995, respectively. He was with ETRI (Electronics and Telecommunication Research Institute) from 1982 to 1991 as a senior technical staff. He also was with KETI (Korea Electronics Technology Institute) from 1991 to 1995 as a senior engineer. Since 1995, he has been with Seokyeong University, Seoul, Korea as a professor. His research areas are in analog and mixed mode IC design for communication, consumer, biomedical and sensor applications. He was the TPC chair of ISOCC'2009 and also was the general chair of ISOCC'2011. 